\title{
LAS CÁTEDRAS CONACYT EN LOS MÁRGENES DE LA SUBCONTRATACIÓN Y LA FLEXIBILIDAD LABORAL
}

Cátedras Conacyt in the margins of subcontracting and labor flexibility

Oyuki Arce Miyaki ${ }^{1}$, Redi Gomis Hernández ${ }^{2}$

Fecha de recepción: 05 de octubre del 2018

Fecha de aceptación: 12 de abril del 2019

1- Nacionalidad: Mexicana. Grado: Maestría. Especialización: Migración. Adscripción: El Colegio de la Frontera Norte. Correo electrónico: oyukidem2016@colef.mx. D ORCID: http://orcid.org/0000-0003-2012-1275

2- Nacionalidad: Mexicana. Grado: Doctorado. Especialización: Ciencias sociales. Adscripción: El Colegio de la Frontera Norte. Correo electrónico: rgomis@colef.mx. ORCID: http://orcid.org/0000-0002-1515-3929 


\section{Resumen}

El objetivo del trabajo es examinar la experiencia laboral de los catedráticos de Conacyt en lo que lleva funcionando el programa. Las Cátedras Conacyt son plazas académicas para jóvenes investigadores bajo un programa novedoso del gobierno mexicano que establece un sistema atípico y flexible de relaciones laborales triangulares para el sector académico en el país. Las condiciones laborales atípicas y flexibles se asocian en buena parte de la literatura con una posible precariedad laboral. En general, este debate está mayormente centrado en los ámbitos de la industria y los servicios, con personal donde prevalecen las bajas calificaciones. Pero este no es el caso de los catedráticos. Por eso vale la pena comprobar si aquí también opera tal asociación. Se realizaron diecisiete entrevistas a jóvenes catedráticos, a partir de las cuales se concluye que, las condiciones laborales de los empleos de Cátedras, como régimen laboral atípico, se percibe positivamente por los involucrados, aunque existen aspectos problemáticos que no deben ser ignorados.

Palabras clave: Cátedras Conacyt, subcontratación laboral, relaciones laborales triangulares, precariedad laboral, flexibilidad.

\section{Abstract}

The aim of this work is to examine the labor practices of Conacyt academic staff from the perspective of their subjective experience in this work field. The "Catedras Conacyt" are academic positions for young researchers, as the result of a new Mexican government program that establishes an atypical and flexible triangular labor relations system for the academic sector in Mexico. In the current debate a relation between the atypical-flexible and the job insecurity is widely accepted, although not everybody agree with this point of view. Overall, this debate is referenced to labor areas of industry and services, usually low-skilled staff, none of which is the case of these young academic staff. So, it is worth checking if that association also operates in Conacyt "Catedras". Seventeen interviews were conducted with young academics, from which it is concluded that hardly can be associated "Catedras" with a condition of precariousness. However, there are problematic aspects that should not be ignored.

Keywords: Catedras Conacyt, labor subcontracting, triangular labor relations, job insecurity, flexibility. 
Revista de Ciencias Sociales y Humanidades. ISSN-P: 0188-9834 ISSN-E:2395-8669.

\section{Introducción}

$\mathrm{E}$

n febrero de 2014 se lanzó en México la convocatoria para las Cátedras Conacyt, plazas académicas destinadas a jóvenes investigadores. A primera vista constituyen empleos favorables para un sector de la población altamente calificado que actualmente encuentra dificultades para insertarse laboralmente en las instituciones académicas: contrato por tiempo indefinido, salarios elevados, prestaciones debidas — seguridad social y de salud, vacaciones pagadas, ahorros para el retiro, entre otras. - , y hasta el derecho al año sabático. Pero tras estas condiciones, indudablemente beneficiosas, también se ha advertido (Gil Antón, 2014) que la relación laboral presenta una distorsión respecto de las normas clásicas de empleos, en tanto transcurren bajo un esquema triangular. Esto es, las instituciones en las que estos jóvenes desarrollan y evalúan toda su actividad de trabajo no son las mismas que las que deciden su ingreso, promoción y permanencia. En efecto, si bien, en las Reglas de Operación de estas Cátedras (CONACYT, 2014a) se plantea que los beneficiarios de tales plazas formarían parte de la plantilla de servicios personales del Conacyt, al mismo tiempo se especifica que, tanto las Instituciones de Educación Superior, como los diferentes Centros de Investigación, fungirían como los ámbitos por excelencia en los que estos jóvenes ejercerían sus funciones en todo su potencial.

Por supuesto que responder para todos los propósitos legales a un empleador mientras que otro es el que dicta los contenidos de trabajo y establece sus reglas de actividad cotidianas podría perfectamente caracterizarse como una condición flexible y atípica de la relación laboral. Este rasgo es lo que caracteriza centralmente a las Cátedras en términos laborales. Y en este sentido, por sus implicaciones tanto teóricas como políticas, la referida triangulación puede llegar a ser motivo de controversia. Aunque de conformidad con los enunciados de Miguélez (2004) y de Reygadas (2011) la flexibilidad y la atipicidad laboral no constituyen propiedades que por fuerza tengan que encerrar una connotación negativa, en una visión menos neutra es usual que la flexibilidad y la atipicidad laboral sean vinculadas cercanamente con la precariedad, siendo esta última una característica que conceptualmente remite a aspectos negativos del empleo (Reygadas, 2011).

Dentro de esta lógica menos neutra, Gil Antón (2014), yendo un paso más lejos, presenta la triangulación de los empleos de Cátedras como un escenario que evoca los regímenes de subcontratación laboral, comparable en ciertos aspectos a los servicios de limpieza o seguridad que actualmente muchas instituciones - tanto del sector público como del privado-, transfieren a terceros. La subcontratación laboral es un caso específico dentro de las condiciones atípicas y flexibles $-\mathrm{y}$ especialmente en estas actividades mencionadas-, al cual la precariedad laboral se asocia con particular fuerza (Dean, 2007; Curiel Sandoval, 2013).

Bajo estas consideraciones, podría entonces preguntarse ¿hasta qué punto los catedráticos Conacyt pueden ser realmente considerados como personal incorporado a un régimen de trabajo subcontratado? Y, de ser este el caso, ¿resultaría entonces justificado suponer que dentro de su situación laboral se encontrarían aspectos relacionados con condiciones de precariedad? Estas son las dos inquietudes principales que surgieron al abordar la problemática de investigación presentada antes. En concordancia con esto, el objetivo de este trabajo es tratar de entender el carácter real de la situación laboral en la que se hallan los investigadores que 
Revista de Ciencias Sociales y Humanidades. ISSN-P: 0188-9834 ISSN-E:2395-8669.

ocupan las cátedras Conacyt, especialmente en los aspectos que sean directamente resultantes del régimen triangular de relaciones contractuales en el que se encuentran inmersos. Este trabajo pretende evaluar en qué medida es válida , la asociación comúnmente aceptada entre flexibilidad y precariedad. Es decir, ¿pueden las Cátedras Conacyt ser al mismo tiempo empleos flexibles pero no precarios?

El tiempo transcurrido desde que comenzó a implementarse el programa y el momento en que se realizó el estudio que se recoge en este artículo, es relativamente corto. Como "experimento" institucional, el programa de Cátedras es un proceso aún en curso. Con toda seguridad se realizarán ajustes a su implementación y, probablemente, también irán apareciendo enmiendas al diseño. Esta inestabilidad del fenómeno en discusión tiene una doble implicación: por una parte, es imposible pretender conclusiones definitivas sobre sus resultados. Las observaciones y análisis deben ser considerados, por tanto, como anticipados, más bien apuntando a la trayectoria perfilada por la situación laboral de los jóvenes catedráticos. Por otra parte, sin renunciar al conocimiento de los aspectos objetivos, se enfatizará la dimensión subjetiva, recuperada a través de las entrevistas realizadas al grupo de estudio. Se busca, sobre todo, entender el fenómeno desde la experiencia de los propios implicados. O sea, cómo perciben los catedráticos su situación laboral en este régimen triangular.

El análisis empírico y las conclusiones se va a apoyar en diecisiete entrevistas realizadas a jóvenes catedráticos en 2016 que, en ese momento, se encontraban trabajando en diez instituciones de doce entidades federativas de la república mexicana. Aunque se deseaba que hubiera existido heterogeneidad por sexos, por ubicación geográfica y por área disciplinaria, en realidad, debido al desconocimiento del conjunto, la selección de los casos estuvo sobre todo fijada por una suerte de bola de nieve.

Programas similares al de Conacyt llevan tiempo implementándose en otros países, como Francia, Argentina y Ecuador. Pero es algo inédito para el sector académico en el contexto mexicano. El trabajo no tiene una perspectiva de políticas públicas. No se busca realizar una evaluación del programa en sí mismo, de sus virtudes o defectos. Y si bien al parecer la implementación de la misma lógica de funcionamiento en los otros países ha tenido resultados positivos, tampoco se busca realizar ahora ningún tipo de comparación.

Luego de esta breve introducción, se desarrollará el trabajo en cinco partes. En la primera, se explica brevemente en qué consisten las Cátedras Conacyt, resaltando los aspectos de mayor interés para los propósitos del trabajo. La segunda, establece las coordenadas conceptuales y teóricas dentro de las que se quiere situar el estudio. La tercera, fija la estrategia metodológica que se siguió para obtener la información empírica. En la cuarta, se realiza el análisis de esa información. Y en la quinta, por último, se avanzan unas conclusiones.

\section{Las Cátedras Conacyt}

En febrero de 2014 se lanzó en México la convocatoria del programa "Cátedras Conacyt" para jóvenes investigadores, las cuales constituyen, en sentido estricto, plazas de carácter académico adscritas al Conacyt. Uno de los requisitos es tener menos de 40 años —en el caso de los hombres-, y menos de 43 - en el de las mujeres-.

El objetivo declarado del programa en la convocatoria fue "incrementar y fortalecer la capacidad de generación, aplicación y transferencia de conocimiento en las áreas prioritarias para el país” (CONACYT, 
Revista de Ciencias Sociales y Humanidades. ISSN-P: 0188-9834 ISSN-E:2395-8669.

2014b: 2), con origen en el Plan Nacional de Desarrollo 2013-2018 (Gobierno de la República, 2013). La inquietud relacionada con la problemática planteada en este trabajo surge en relación con la modalidad planteada para alcanzar ese objetivo. En la misma convocatoria se establece que se pretende lograrlo "mediante la incorporación de investigadores comisionados [por Conacyt] a las instituciones y entidades [en México] que realizan investigación y formación de capital humano" (CONACYT, 2014b: 2). En esencia se tratarían de plazas académicas colocadas dentro una relación laboral con un sesgo inusual en el ámbito académico mexicano, de acuerdo a Gil Antón (2014). Esto es, serían trabajadores de Conacyt, pero comisionados a realizar su trabajo habitual en otras instituciones.

Hay un doble contrato por parte de Conacyt derivado del formato, ambos de naturaleza diferente. Por una parte, está el "Nombramiento", que constituye el instrumento jurídico que expide Conacyt a los investigadores y tecnólogos que hayan sido seleccionados para ocupar las plazas académicas de Conacyt (CONACYT, 2014a: 14). Conacyt fungirá como su único patrón y, por lo mismo, será el responsable de las prestaciones laborales, sueldos y seguridad social de los contratados. En términos laborales, las instituciones beneficiadas no establecen ningún compromiso directo de carácter legal con los catedráticos. Pero sí con Conacyt. Entre este y las instituciones beneficiadas se firman contratos interinstitucionales llamados "Convenios de Asignación de Recursos Humanos" (CONACYT, 2014a: 5-6). Es el instrumento jurídico mediante el cual se formalizan las condiciones de la comisión de las Cátedras, así como los derechos y obligaciones de las instituciones involucradas. El contrato de nombramiento es el mismo para todos los catedráticos, pero los convenios con las instituciones son específicos y, por tanto, diferentes entre sí. Este es un elemento importante a tener en cuenta.

Con una base anual, desde 2014 se han completado totalmente tres convocatorias, y una cuarta se encuentra en curso. De acuerdo a los datos del portal en línea de Conacyt (http://conacyt.gob.mx/index. $\mathrm{php/convocatorias-conacyt/convocatorias-conacyt/convocatoria-catedras/padron-de-beneficiarios-ca-}$ tedras-conacyt) hasta ahora se han autorizado unas 1400 de estas Cátedras y 108 instituciones han sido beneficiadas por el programa. Los hombres predominan por sobre las mujeres (58.2\% versus $40.9 \%$ ); la temática más beneficiada de los proyectos aprobados corresponde a "desarrollo tecnológico" (25.8\%), mientras que la menos destacado ha sido "conocimiento del universo" (3.8\%); aunque la intención del programas ha sido equilibrar regionalmente las comisiones, se sigue favoreciendo una distribución centralista de los recursos: el 5.5\% de las instituciones — todas ubicadas en el centro del país, principalmente en la Ciudad de México-, ha recibido el 30.2\% de los catedráticos. El 57.4\% de las instituciones que lograron acceder a los beneficios del programa, en conjunto, concentran únicamente el $19.7 \%$ de las plazas. Entre las instituciones más apoyadas por el sistema de Cátedras se encuentran la Universidad Nacional Autónoma de México (UNAM), el Tecnológico Nacional de México (DGEST), el Instituto Politécnico Nacional (IPN), la Universidad Autónoma de San Luis Potosí, el Centro de Investigación y de Estudios Avanzados del IPN y la Universidad Autónoma Metropolitana.

En los Lineamientos se explica de manera más detallada el modo de operación del programa. Hay cinco aspectos cuyas particularidades distintivas consideramos importante en función del trabajo: la naturaleza de la relación contractual, la disociación del lugar de trabajo, la duración de la relación laboral y las condiciones de trabajo. A fin de contextualizar los resultados, todas ellas se irán tratando en detalle a medida que se vayan analizando. 
Revista de Ciencias Sociales y Humanidades. ISSN-P: 0188-9834 ISSN-E:2395-8669.

\section{Subcontratación y precariedad: revisión teórica}

La subcontratación y la precariedad, desde una perspectiva laboral, son los conceptos centrales en torno a los cuales se encuentra estructurado el presente estudio. En este apartado se busca recuperar al menos parte de la discusión sobre las complejas realidades y situaciones a las que tales términos remiten, con la promesa de que ello contribuya a brindarnos una representación más cabal de las cuestiones de interés particular.

\subsection{La subcontratación laboral}

El término "subcontratación" se emplea usualmente para designar a un conjunto más o menos amplio de realidades relativamente heterogéneas entre sí (OIT, 1997; 1998; 2006; Bensusán, 2007), aunque con una identidad común: aluden a situaciones que involucran la externalización de actividades y funciones que previamente se realizaban de manera interna. Ello como parte de una estrategia de fragmentación que supuestamente le permitiría a las firmas concentrarse en sus competencias centrales, dejando al mismo tiempo en manos de proveedores la solución de aquellas otras tareas consideradas periféricas (Echeverría Tortello, 2010).

Para fines de este estudio no es relevante la subcontratación comercial, en la que se encarga a las terceras partes es la producción de algún bien o la prestación de algún servicio. Pero sí se retoma el concepto de subcontratación laboral, cuyo objetivo único, o predominante, es el suministro externo de mano de obra, que si bien en sentido estricto también calificaría como un servicio - en este caso de "colocación" (Echeverría Tortello, 2010: 62) - , hay dos diferencias importantes de este arreglo respecto del comercial: (1) el pago a los subcontratistas va a depender de la cantidad y la calidad de trabajadores "servidos" (OIT, 1997); (2) las personas subcontratadas están sujetas al control y la supervisión directa de la empresa usuaria o subcontratante (OIT, 1997).

Bajo esta óptica, el "trabajo en régimen de subcontratación" constituye un concepto clave (OIT, 1997; 1998). En esencia, hace referencia a las relaciones entre trabajadores subcontratados — directamente o a través de intermediarios- y una empresa usuaria de los mismos, de la cual no son empleados formales, pero para la cual - y/o en la cual-desarrollan su trabajo (OIT, 1997: 6). Hay tres elementos relevantes en este enunciado que vale la pena rescatar (Bensusán, 2007; Echeverría Tortello, 2010): (1) en términos formales, las relaciones entre los trabajadores subcontratados respecto a la empresa usuaria se caracterizan por la independencia y autonomía legal; (2) en la práctica, sin embargo, los trabajadores subcontratados se encuentran en condiciones de dependencia respecto a la empresa usuaria; (3) las relaciones entre las dos partes mencionadas pueden establecerse directamente - con supuestos trabajadores por cuenta propia-, o indirectamente - a través de agentes suministradores externos, que ponen a disposición de las empresas usuarias a trabajadores previamente contratados por ellos mismos-.

Las referencias a la subcontratación laboral remiten casi invariablemente a la "relación de trabajo triangular", que "surge del hecho de que los trabajadores de una persona (el 'proveedor' o 'suministrador') trabajan para una tercera persona (el 'utilizador' o 'usuario')" (OIT, 2006: 14). Un rasgo característico de 
Revista de Ciencias Sociales y Humanidades. ISSN-P: 0188-9834 ISSN-E:2395-8669.

las formas triangulares de subcontratación es que se celebren dos contratos. Uno de carácter comercial entre el proveedor y el usuario, en el que se estipularía las condiciones del intercambio entre ellas. El otro de naturaleza laboral entre el proveedor y el trabajador, cuyo objetivo es establecer las condiciones de trabajo (Bensusán, 2007).

El trabajo en régimen de subcontratación, en general, y las relaciones de trabajo triangulares, en particular, no constituyen problemáticas exclusivas del ámbito de las empresas, aún cuando su uso en la literatura se encuentre situado con más fuerza en los contextos empresariales. A nuestro juicio, el régimen de Cátedras representa un caso de subcontratación laboral de tipo triangular. Tal interpretación se ajusta en gran medida a los enunciados y descripciones presentados antes: (1) en cuanto a los agentes involucrados, la triangulación quedaría plenamente establecida entre Conacyt (como agente suministrador), las Instituciones de Educación Superior y los Centros de Investigación (como agentes usuarios o "instituciones beneficiadas", según la terminología empleada por el propio Conacyt) y los catedráticos o "personal académico de Conacyt" (como trabajadores en régimen de subcontratación). Y esto, sin importar que ninguna empresa esté involucrada en el arreglo. Todos los agentes son instituciones públicas; (2) en cuanto a la naturaleza de los pactos refrendados se fijan dos figuras contractuales, ambas firmadas por Conacyt. Una suscrita con las instituciones beneficiadas. La otra con los candidatos seleccionados. Y aunque toda la actividad laboral de los catedráticos es realizada en el recinto de las instituciones académicas beneficiadas, no hay espacio en las reglas para acuerdos formales entre estos dos agentes, a pesar de que probablemente sea el subconjunto del sistema en el que tengan lugar las interacciones más continuas e intensas.

Lo anterior resulta interesante, en todo caso, porque es una iniciativa que proviene del propio Estado, en tanto constituye, como plantea Bronstein (1990), "un gran productor de formas flexibles de empleo" (pág. 406). En general, parte del debate acerca de la subcontratación laboral tiende a enfatizar sus implicaciones negativas, siendo una de las imputaciones más socorridas convertirla en un fundamento casi inequívoco de la precariedad laboral, aún cuando la realidad no siempre se comporte bajo esta lógica, como suponemos que es el caso de los empleos de Cátedras. Pero antes de examinar la relación entre estos dos fenómenos, examinemos primero el significado del concepto de precariedad laboral.

\subsection{La precariedad laboral}

Por regla general, la conceptualización de la precariedad se formaliza por defecto. Calificar a un empleo como precario siempre supone una referencia, ya sea explícita o implícita, a una norma o estándar. Con mayor o menor grado de coincidencia, en general se considera que la mención o la alusión al empleo estándar tienen como referentes trabajos con las siguientes características: (1) de tiempo completo, de duración indeterminada, sin ininterrupciones: (2) con un único empleador, con un horario fijo, sin jornadas extenuantes, etc.; (3) con ingresos constantes y suficientes; (4) con un plan de pensiones, protección en caso de enfermedad y desempleo, con garantías legales o colectivas que lo garanticen, etc. (Rodgers, 1989; ESOPE, 2005; Bensusán, 2007; Reygadas, 2011; Guadarrama, Hualde y López, 2016).

Un elemento importante a destacar del concepto de precariedad es que ese estándar al que remite no es un parámetro neutro, y es dudoso que constituya un referente empírico (ESOPE, 2005; Reygadas, 2011). Según ESOPE (2005) constituye ante todo un referente esencialmente normativo, un "estándar de 
Revista de Ciencias Sociales y Humanidades. ISSN-P: 0188-9834 ISSN-E:2395-8669.

aceptabilidad", que estaría condensando una representación considerada adecuada del empleo "de calidad, "digno", "decente". De manera que el empleo precario, por su parte, frente a esto no encarnaría sino una categoría con una connotación negativa, una suerte de "subestándar" (ESOPE, 2005: 47),

Por otra parte, es ampliamente aceptada la idea de que la precariedad constituye una condición multidimensionalmente determinada (Cano, 1998; ESOPE, 2005; Diana Menéndez, 2013; Guadarrama et al., 2016; Mora Salas, 2012). En el trabajo se va a asumir la propuesta de Guadarrama et al. (2016). Las dimensiones que a juicio de estos autores se deben tomar en cuenta son: (1) la temporal, referido al grado de certidumbre sobre la continuidad o permanencia en el empleo; (2) la organizacional, que tiene que ver con el grado de control sobre las condiciones de trabajo: condiciones de trabajo (horarios, intensidad, seguridad); (3) la económica, relacionada con el ingreso: suficiencia; estabilidad; estrategia de empleos múltiples; (4) la social, que hace referencia al grado de protección ya sea jurídica, colectiva o consuetudinario: pertenencia a asociaciones, seguridad social (seguro); (5) la subjetiva, que tiene que ver con el grado de satisfacción, percepciones, vivencias, identidad, etc.

Esta proposición incorpora las mismas cuatro dimensiones sugeridas por Rodgers (1989) y retomadas luego por ESOPE (2005). Pero añade a aquellas la dimensión subjetiva, que según los mismos autores es frecuentemente ignorada en el análisis del fenómeno. Y este es justamente uno de los aspectos que el presente estudio intenta rescatar.

Una última cuestión es acerca de la relación entre la condición estándar y la condición precaria. Se pueden vislumbrar, al menos a nivel teórico, tres modelos o representaciones de las relaciones entre ambos componentes de la ecuación: (1) dual; (2) de grados; (3) de clases.

El modelo dual o dicotómico supone una visión del empleo sobre un eje con dos valores. La dificultad teórica y metodológica es determinar el punto de giro en que una condición cede lugar a la otra. El modelo de grados de precariedad es el más aceptado por los autores examinados (Rodgers, 1989; Cano, 1998; ESOPE, 2005; Diana Menéndez, 2013; Guadarrama et al., 2016). Sin negar la dualidad que reconoce la existencia de empleos dignos y precarios, quienes sostienen esta otra lógica plantean que es más útil un enfoque que considere la precariedad como un continuo dentro del cual se podrían advertir grados o niveles en lugar de concebirlos como estados dicotómicos. Los diferentes grados de precariedad se identificarían por alguna combinación particular de estos factores, lo cual inevitablemente establecería limites cambiantes, y en alguna medida arbitrarios, en torno al concepto. El tercer modelo posible, el de clases de precariedad, es atribuido a Bouffartigue (2008 citado por Diana Menéndez, 2013). Este autor distingue entre tres clases o tipos de precariedad, a su parecer distintos entre sí. Estos son: (1) la precariedad del empleo, sujeto a las características de los contratos; (2) la precariedad del trabajo, vinculada al deterioro de las condiciones laborales; y (3) la precariedad de relaciones laborales, que tiene que ver con la ineficacia o la débil aplicación del derecho del trabajo y del derecho sindical. Estas tres clases de precariedad pueden darse, según Bouffartigue, aisladamente o de manera combinada y superpuesta. La idea fundamental en relación a este modelo es que la realidad compleja a la que remite el fenómeno de la precariedad no tiene que ser reducida a una única categoría lingüística. No hay por fuerza una sola condición de precariedad, aunque varíe a lo largo de una escala, sino que puede representar una categoría heterogénea, con múltiples formas de manifestarse. Es probable que justamente esto hayan tenido en mente Guadarrama et al. (2016) cuando hablan de "zonas grises" de empleo, en las que "el balance de las situaciones no es tan claro en la medida en que determinadas dimensiones del trabajo pueden evolucio- 
Revista de Ciencias Sociales y Humanidades. ISSN-P: 0188-9834 ISSN-E:2395-8669.

nar de manera favorable y, otras, mantener rasgos precarios muy acusados" (p. 22).

\subsection{El trabajo en régimen de subcontratación y la precariedad laboral}

Se pueden advertir dos vertientes respecto de juzgar a la subcontratación laboral como fuente de precariedad. Una es de carácter general, y en esta la precariedad supuestamente derivaría del carácter atípico y flexible de la subcontratación. La otra es más específica, y trata de establecer las maneras concretas en las que las relaciones de trabajo establecidas por los actores en régimen de subcontratación afectan dimensiones específicas de su situación laboral asociadas con el concepto de precariedad.

En cuanto a la primera vertiente todos los autores revisados aceptan que la subcontratación laboral, en general, y en particular la modalidad triangular, pueden ser catalogadas formas tanto atípicas como flexibles de relación laboral. Lo atípico considerado como algo novedoso, una situación que antes no existía y que surge como resultado natural del desarrollo (ESOPE, 2005; Reygadas, 2011). Lo flexible, por su parte, como una oposición intencional a rigideces legales y esquemas entumecidos de prácticas de los mercados de trabajo (Bronstein, 1990).

Es muy común encontrar que los trabajos atípicos y los trabajos flexibles sean al mismo tiempo empleos precarios (Rodgers, 1989; Bronstein, 1990; Miguélez, 2004; ESOPE, 2005; Reygadas, 2011), pero no ocurre así en todos los casos. Aunque concurrentes, las relaciones entre los desarrollos atípicos y precarios son complejas: pueden determinarse unívocamente, de manera recíproca o pueden coexistir independientemente (Reygadas, 2011). Todo lo anterior pudiera perfectamente hacerse extensivo a las relaciones que guarda la precariedad con la flexibilidad. De manera concreta, en lo relativo al objeto de estudio, significa que esta flexibilidad laboral podría teóricamente estar vinculada a un situación de precariedad, como tal experimentada por los catedráticos. Pero aún así, no debería asumirse mecánicamente que tal condición derivaría por fuerza de la contratación laboral de tipo triangular de los catedráticos.

En relación a la segunda vertiente, destacaremos algunas condiciones negativas específicas mencionadas en la literatura que supuestamente se desprenderían como un resultado directo de la relación laboral de subcontratación. Podrían ser importantes para entender la situación en la que se encuentran los catedráticos. No se presentan en un orden jerárquico de importancia.

Uno: se presenta debilitamiento de la protección colectiva. Este es, de acuerdo a Ermida Uriarte y Colotuzzo (2009), el principal problema que plantea la subcontratación al movimiento sindical. Tres cuestiones a mencionar en este aspecto: (1) fragmentación que dificulta la organización de las fuerzas dispersas; (2) desintegración respecto de los trabajadores titulares de la empresa beneficiaria, lo que también podría afectar la fuerza de los sindicatos internos, por eventual papel de esquiroles; (3) imposibilidad en el caso de los catedráticos por su nombramiento como personal de confianza.

Dos: puede haber falta de vigilancia sobre algunas condiciones de trabajo. Por lo general el empleador se mantiene, como señala Echeverría Tortello (1997), "completamente fuera del escenario material donde se realiza el trabajo” (p. 18). Es difícil en estas condiciones para el empleador mantener una cuidadosa inspección sobre el cumplimiento por parte de la institución beneficiaria de los contratos suscritos con 
Revista de Ciencias Sociales y Humanidades. ISSN-P: 0188-9834 ISSN-E:2395-8669.

ella respecto de la mano de obra subcontratada (Ermida Uriarte y Colotuzzo, 2009). Esto podría generar cierta desprotección frente a acciones arbitrarias de discriminación ante las cuales se carecería, además, de defensa colectiva.

Tres: confusión respecto de la figura responsable. En ciertos aspectos puede desdibujarse cuál es específicamente el nivel de responsabilidad que le toca a cada uno de los "patrones" para garantizar su cumplimiento. La resolución de algunas controversias puede quedar comprometida en tanto caerían en terreno de nadie (Echeverría Tortello, 1997; Ermida Uriarte y Colotuzzo, 2009).

Cuatro: no hay estabilidad laboral y existe incertidumbre en cuanto a la continuidad del trabajo (Echeverría Tortello, 1997; Bensusán, 2007). Por lo regular en los esquemas de subcontratación triangulares las entidades intermediarias son agencias de colocación de trabajo temporal, respondiendo a las oscilaciones de las solicitudes por parte de las empresas que demandan el servicio. Por eso casi siempre los contratos de trabajo ofrecidos a los trabajadores por parte de las contratistas son, además, de carácter temporal.

Cinco: puede conducir a actividades ilegales encubiertas. Esta es la consecuencia más frecuentemente aludida. Hay dos formas de ilegalidad reportadas. La primera sería la de ocultamiento de la identidad del empleador que lo liberaría de tener que aparecer como parte directa en la relación de trabajo, despojándose con ello de la necesidad de asumir esa responsabilidad. La segunda, puede conducir a situaciones de encubrimiento de una relación de trabajo. Sería un caso de violación dentro de la subcontratación directa, por la cual se persuade al trabajador que preste sus servicios como "igual independiente" mediante un contrato comercial, cuando en realidad mantiene una relación subordinada esencialmente de carácter laboral. De cualquier manera, estas formas de proceder fraudulentas reducen la protección de los trabajadores y deprimen sus condiciones laborales (Añez Hernández, 2012).

\section{Estrategia metodológica y datos empíricos}

Para obtener información empírica a fin de examinar y responder a la problemática de interés, se realizaron entrevistas semiestructuradas a jóvenes incorporados a las "Cátedras Conacyt". En total se realizaron 17 entrevistas de catedráticos comisionados a 6 instituciones académicas del país. Aunque el número de entrevistados no es representativo para realizar generalizaciones sobre el tema, tampoco es lo que se persigue. Por ello consideramos que la multiplicidad de información obtenida con los casos estudiados debe resultar suficiente para lograr captar la complejidad asociada a la problemática de interés. Las entrevistas tuvieron como objetivo indagar sobre la experiencia subjetiva de los entrevistados en diferentes áreas de su vida laboral desde que comenzaron en su condición laboral actual. Constituye, por supuesto, una evaluación de corto plazo, tomando en cuenta que al momento de las entrevistas todo el programa llevaba entonces apenas dos años de vida desde que comenzara a implementarse.

Aunque se parte de asumir que la precariedad es una condición habitual de todo empleo, al menos en algún grado, es importante señalar que metodológicamente es complicado definir, ya sea el punto exacto de quiebre que distingue a un empleo precario de otro que no lo es, ya sea el grado preciso de precariedad que el mismo incorpora. Y, por supuesto, se necesitaban datos empíricos para poder evaluar y determinar hasta qué punto los empleos de Cátedras tenían rasgos de precariedad, así fuera desde el punto de vista subjetivo. Es por ello que en las entrevistas se buscaba indagar vivencias experimentadas 
Revista de Ciencias Sociales y Humanidades. ISSN-P: 0188-9834 ISSN-E:2395-8669.

por los catedráticos en los distintos elementos presentes en las dimensiones de la precariedad por separado, similar a lo que en su momento hizo ESOPE (ESOPE, 2005). Fundamentalmente se tratarán de determinar los aspectos derivados del régimen laboral que constituyan para los catedráticos fuente de dudas, incertidumbres y desasosiegos.

En general, los temas que conformaron la guía de entrevista iban desde los datos sociodemográficos hasta las dinámicas familiares, pasando por el contrato, la comunicación con compañeros de trabajo y superiores, los salario y prestaciones, las experiencias respecto a movilidad por motivo laboral, el sentido de pertenencia, etc. Todo ello con el fin de poder vincular las experiencias en su trayectoria laboral y de vida, con la percepción que en ese momento tenían sobre las condiciones laborales.

\section{Resultados}

El cuadro 1 presenta un perfil general de los diecisiete catedráticos seleccionados para entrevista, por lo que no nos detendremos en su caracterización.

Cuadro 1. Perfil de los catedráticos entrevistados $(\mathrm{n}=17)$

\begin{tabular}{|c|c|c|}
\hline \multirow{2}{*}{ Característica } & Descripción & Valor \\
\hline \multirow{2}{*}{ Sexo } & Mujeres & 5 \\
& Hombres & 12 \\
\hline Edades & Rango & $32-40$ años \\
\hline \multirow{2}{*}{ Estado civil } & Casados & 10 \\
& Solteros & 5 \\
& Unión libre & 2 \\
\hline \multirow{3}{*}{ Localización de las instituciones } & Baja California & 2 \\
& Guerrero & 2 \\
& Campeche, Chetumal, Chihuahua, Ja- & 1 \\
\hline \multirow{2}{*}{ Movilidad geográfica comisión } & Oaxco, Michoacán, Nayarit, Nuevo León, & 15 \\
\hline \multirow{2}{*}{ Origen nacional } & Foráneos & 2 \\
\hline \multirow{2}{*}{ Generación Cátedras } & Locales & 14 \\
& Mexicanos & 3 \\
\hline \multirow{2}{*}{ Temas } & Extranjeros & 13 \\
& Primera & 4 \\
\hline \multirow{2}{*}{ Modalidad } & Segunda & 6 \\
& Desarrollo tecnológico & 6 \\
\hline & Sociedad & 2 \\
\hline & Ambiente & 2 \\
\hline & Conocimiento del universo & 12 \\
\hline & Energía & 5 \\
\hline
\end{tabular}

Fuente: (Arce Miyaki, 2016) [Entrevistas a catedráticos del programa Cátedras Conacyt] 
Revista de Ciencias Sociales y Humanidades. ISSN-P: 0188-9834 ISSN-E:2395-8669.

A continuación, se presentan los principales hallazgos. Ante todo, debe decirse que, aunque la evaluación del proyecto de Cátedras no haya sido uno de los objetivos primarios del trabajo, es inevitable la referencia a la visión de conjunto que tienen los entrevistados sobre su experiencia en Cátedras (cuadro 2). Llama la atención que todos los entrevistados, sin excepción, tienen una valoración general positiva del programa. Algunos, como es natural, más que otros. Expresiones similares a "soy de las personas que $[. .$.$] cree que fue una política pública muy buena", "es una de las mejores opciones que ha habido en$ muchos años para investigadores jóvenes" o "yo creo que fue lo mejor que me pudo haber pasado" fueron comunes en las entrevistas.

\section{Cuadro 2. Experiencia general de los catedráticos respecto del programa en su conjunto}

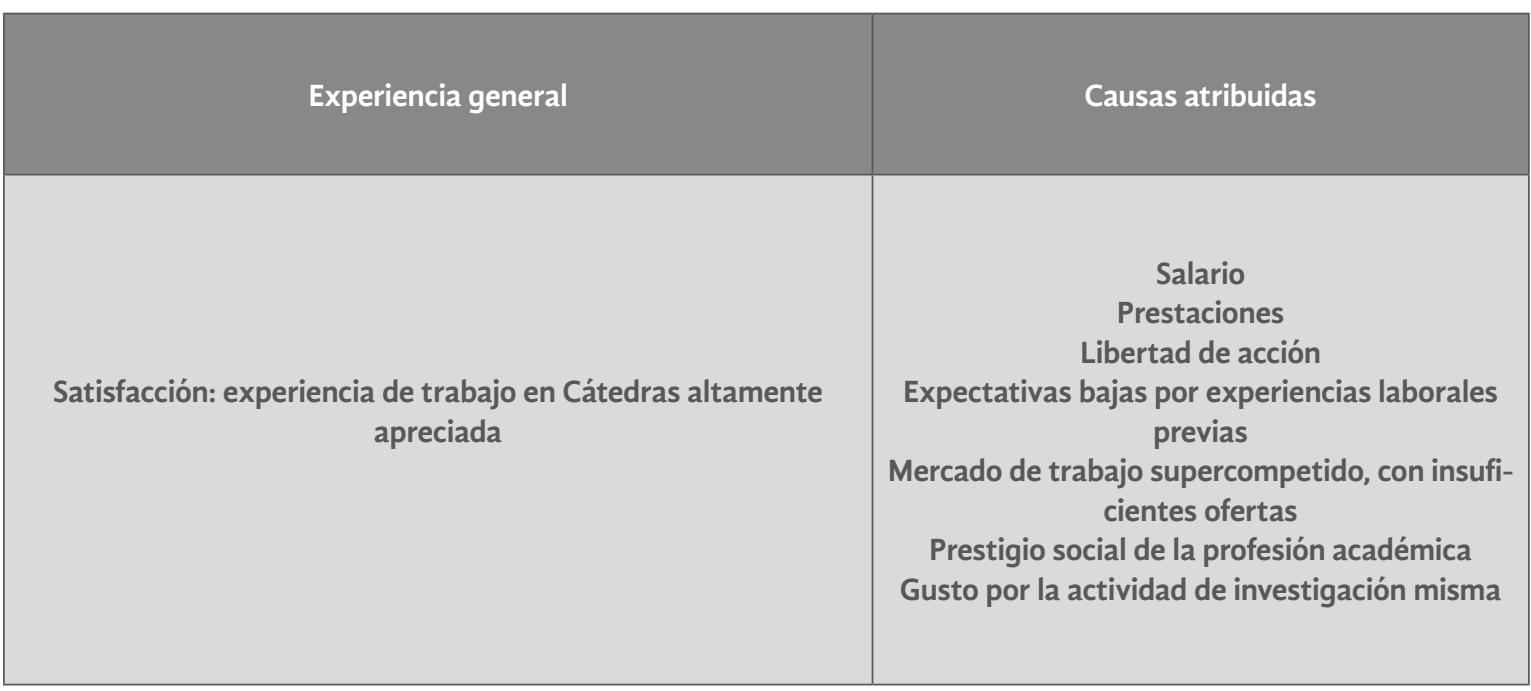

Fuente: Elaboración propia

El salario y las prestaciones incorporadas al puesto fueron las dos principales fuentes de satisfacción mencionadas. Pero en general tiene que ver con condiciones de trabajo favorables. La mayoría de éstas se resumen en el "Estatuto del personal académico del Conacyt", que se encuentra como anexo al documento que contiene los Lineamientos. Por una parte, allí se detallan las prestaciones que tendrían por convenio los catedráticos: salarios elevados (superiores a los de los investigadores de planta en las instituciones receptoras de los catedráticos); vacaciones pagadas (dos periodos vacacionales de diez días cada uno) y prima vacacional; derecho a licencias (con goce y sin goce de sueldo); aguinaldo (cuarenta días de sueldo); fondo de ahorro; año sabático; atención médica ISSSTE. Además, un conjunto de otras prestaciones relativamente menores como prima de antigüedad, seguro de vida institucional, seguro de retiro, material didáctico, anteojos, etc. Por otra parte, un aspecto a destacar en relación con las condiciones laborales se recoge en el artículo 26 de los Lineamientos, en el que se expresa que es responsabilidad de la institución beneficiada asegurar que los catedráticos gocen de igualdad de condiciones que el de planta en cuanto al acceso a la infraestructura, el equipamiento y los servicios necesarios para el desarrollo de sus tareas. 
Revista de Ciencias Sociales y Humanidades. ISSN-P: 0188-9834 ISSN-E:2395-8669.

En general, las condiciones laborales fijadas en el contrato de Cátedras superaban con creces las disminuidas expectativas que la inmensa mayoría de los entrevistados traía consigo, generadas a partir de inciertas trayectorias laborales previas - la mayoría, incluso, en el ámbito académico-, en las que prevalecían los contratos temporales, consultorías, clases por asignatura y, en el mejor de los casos, becas de posdoctorado. Y es que, en efecto, también los mercados de trabajo académicos se han venido flexibilizando - con descensos en los niveles salariales y degradación de algunas condiciones laborales-, desde los ochenta del siglo pasado en todo el mundo como resultado de presiones políticas y económicas de carácter neoliberal (Slaughter y Leslie, 2001; Llomovatte y Wischnevsky, 2012; Acosta Reveles y Aguilar Torres, 2014). Uno de ellos lo describe así: "Ya me sentía como muy..., como muy inestable. Estaba muy cansada como de agarrar muchas chambitas que [aunque] me dejaban mucho dinero $[. .$.$] eran todas inestables".$

Por supuesto que los catedráticos entrevistados reconocieron la existencia de problemas, como se verá seguidamente, pero los beneficios percibidos exceden ampliamente los inconvenientes o desventajas que pudieran haber señalado. $\mathrm{Y}$, en general, muchos reconocen que una buena parte de las problemáticas enfrentadas tienen que ver con lo novedoso del programa, aunque al mismo tiempo guardan la esperanza de que a medida que surjan los problemas y los mismos sean reportados y resueltos, se generarán mecanismos que contribuirán, si no a evitarlos, al menos a facilitar su solución en el futuro.

"Es cierto que a veces uno se queja o uno exige cosas. Pero también hay que ir dando flexibilidad. Poco a poco..., porque la cosa es nueva. Tampoco vamos a tener los resultados así..., de la noche a la mañana. $\mathrm{Y}$ creo que en base a retroalimentaciones y comentarios de nosotros - positivamente hablando, críticas constructivas-, se va a ir mejorando todo esto".

Hay problemas, sin embargo, que independientemente de esta presunción de que se irían solucionado como parte del aprendizaje institucional progresivo, al parecer han generado niveles importantes de incertidumbre entre los catedráticos. En lo que sigue, examinaremos las tres áreas de problemas que consideramos centrales desde la perspectiva del abordaje que ha prevalecido en este documento. Estas son: proceso de selección, duración o grado de estabilidad del empleo e integración a las instituciones (cuadro 3).

\section{Cuadro 3. Principales áreas laborales experimentadas como problemáticas por los catedrático}

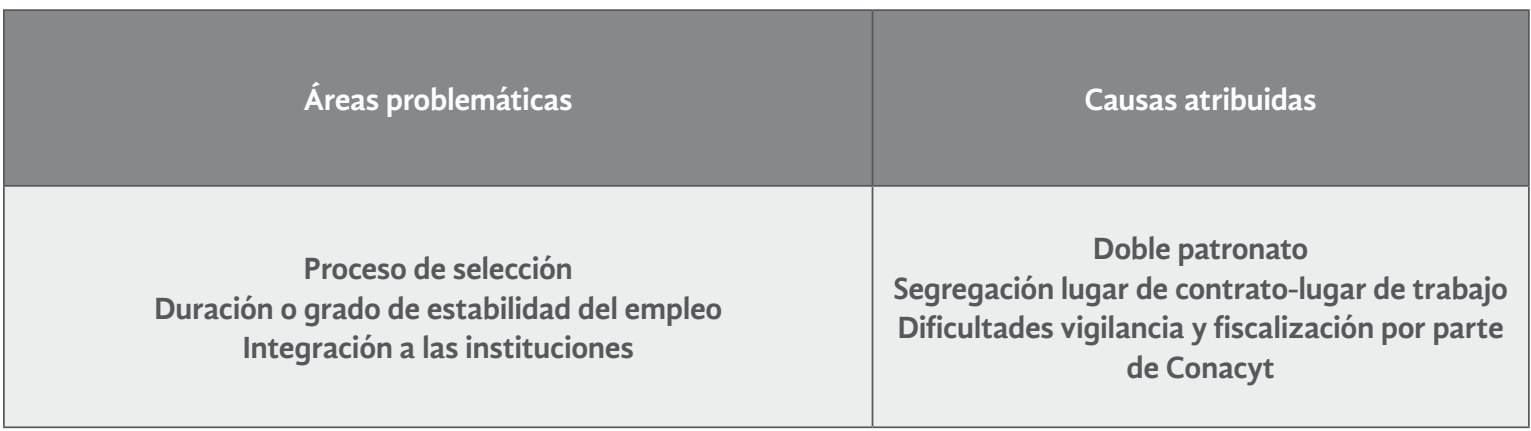

Fuente: Elaboración propia 
Revista de Ciencias Sociales y Humanidades. ISSN-P: 0188-9834 ISSN-E:2395-8669.

\subsection{La selección de los aspirantes: proceso indirecto e incierto}

Hay dos rasgos del proceso de selección con huella en los catedráticos. En primer lugar, no es directa. Las convocatorias no constituyen concursos para elegir al mejor postulante, y sólo en parte la elección depende de los méritos personales de los contendientes. Es resultado de un proceso reglamentariamente complejo. Cada convocatoria es de doble carril. Una dirigida a los jóvenes aspirantes a las plazas, quienes entran al sistema su perfil y relación de intereses, a fin de conformar un padrón o cantera. La otra destinada a las instituciones que aspiran ser beneficiadas con la comisión de dichas plazas, las cuales deben presentar proyectos de investigación, que constituyen al cabo los principales oponentes en la contienda. Y, por eso, lo primero que se eligen son proyectos. Las instituciones ganadoras presentan a Conacyt una terna de entre los aspirantes dados de alta en el padrón por cada Cátedra pretendida. Conacyt decide a quién otorgaría el nombramiento y que sería comisionado a la institución beneficiada. Pero únicamente, en principio, para realizar aquellas tareas requeridas como parte del proyecto favorecido. Para que la selección se materialice es requisito que se logre previamente este match o empate, conjuntamente con el consentimiento de las partes involucradas, entre institución, proyecto y joven aspirante.

Uno de los principios más importantes en la academia ha sido siempre la libertad de cátedras, bajo cuya lógica el investigador desarrollaría su propio proyecto de investigación. En la racionalidad del proceso de contratación practicado por Cátedras hay una suerte de lógica invertida. Se selecciona primero al proyecto y se busca a un investigador afín que lo desarrolle. Como señala un entrevistado: “iY qué significa [para el programa] que todo funcione? Significa que tú, como investigador, te desarrollas plenamente dentro de un proyecto que otra persona escribió". Se podría suponer que por la manera en que fue concebido y funciona el programa, desaparece algo tan sagrado como la libertad de cátedra. Sin embargo, no fue lo que se encontró en la investigación. Uno de los elementos más valorados del programa tiene que ver curiosamente con lo que varios entrevistados coinciden en llamar "la libertad para investigar". Aunque los procedimientos internos de cada institución beneficiada van a poner un sello propio sobre las dinámicas de trabajo emprendidas por los catedráticos, al parecer no se les impide desarrollar propuestas personales, siempre y cuando cumplan satisfactoriamente con las tareas emanadas del proyecto institucional para el cual fueron comisionados. En este sentido, uno de los entrevistados señalaba que: "Tenemos muchas libertades. O sea..., [en cuanto al trabajo en el proyecto] haz de cuenta que eso me toma como el cuarenta por ciento de mi tiempo de trabajo. El otro sesenta yo puedo hacer lo que me dé la gana con cualquier otro investigador. O sea..., ahí si te digo..., no tengo que pedirle permiso [al jefe del proyecto ni al enlace institucional] ni a quién sabe quién en Conacyt".

La complejidad del proceso y el escaso control sobre el mismo en la mayoría de los aspirantes, puede ser un factor generador de dudas, incertidumbres y aprensiones. Este es el segundo rasgo del proceso de selección mencionado al inicio del epígrafe. Partiendo de las experiencias narradas por los entrevistados, se pueden identificar dos tipos de "empates" entre aspirantes y proyecto: (1) ciego, donde el match se va perfilando en el transcurso del proceso; (2) inducido, en el que las especificaciones de los requerimientos de las propuestas institucionales están armonizadas de antemano con las características de aspirantes previamente escogidos. Ambos tipos de selecciones son vividas de maneras muy diferentes por los aspirantes. 
Revista de Ciencias Sociales y Humanidades. ISSN-P: 0188-9834 ISSN-E:2395-8669.

Por un lado, aquellos que participaron en desarrollos concertados parecen haber sentido en todo momento confianza en los resultados. Las dudas son insignificantes. Uno de los entrevistados manifestó que al inscribirse al padrón de aspirantes le pedían describir hasta tres líneas temáticas en las que le gustaría participar de ser eventualmente escogido. Pero que únicamente puso una línea, aquella a la que con casi total seguridad suponía que lo asignarían, porque ya estaba trabajando para el proyecto y sabía que la institución lo propondría en primer lugar: "Como que de antemano yo ya sabía que... Es como cuando vas a una entrevista de trabajo y ya sabes que sí te lo van a dar. Ya ni sientes ningún estrés ni nada, porque sabes que es para ti". Naturalmente, dada la lógica del proceso existe siempre algún nivel de incertidumbre en este proceso, incluso para las instituciones. La decisión final de quién será contratado entre la tercia propuesta es de Conacyt y es, además, inapelable (CONACYT, 2014c: 5). Y, aunque tal vez improbable, esa decisión final puede en teoría no corresponder a lo esperable ni por las instituciones ni por los aspirantes, en función de lo acordado en principio entre ellos.

Pero, por otro lado, este no es el caso de los que siguen un proceso menos concertado, por cuya razón su curso no es tan obvio o previsto, ni para los aspirantes ni para las instituciones. Por su naturaleza, es esquivo para todos los participantes y, lógicamente, genera mayor incertidumbre. Así relata un entrevistado su experiencia al respecto:

Ahí aplicas ciegamente, ¿no? [...] Es un poco como estas páginas de Internet que juntan a las parejas, como match.com, o esas... [...] Entonces tu metes tu perfil sin saber quién está del otro lado. O sea, yo antes no sabía que [esta institución] estaba buscando Cátedras [...] Ya después tú te das cuenta, porque [los funcionarios de Conacyt] te mandan un correo de qué instituciones están interesadas en ti.

4.2. Nombramiento y permanencia: ¿estabilidad temporal comprometida?

A pesar de constituir nombramientos por tiempo indefinido, según consta explícitamente en el contrato de trabajo, resulta un área en la que los entrevistados expresaron cierto nivel de incertidumbre. Y esto, en dos sentidos: (1) en cuanto a la estabilidad del programa como tal; es decir, que por alguna razón el mismo no pueda sostenerse, ya no a largo plazo, sino ni siquiera a mediano plazo; (2) en cuanto a poder permanecer realmente en el programa, de no ser separado del mismo.

Hay un punto en el contrato que constituye para algunos una fuente importante de desconfianza y temor sobre la persistencia del programa. En la cláusula sexta se plantea que el "nombramiento" se encuentra sujeto a las disponibilidades presupuestales determinadas por la Secretaría de Hacienda y Crédito Público. Por cuya razón se advierte que, en caso de que la partida presupuestal correspondiente sea eliminada, el Conacyt podrá dejar sin efecto tal nombramiento "sin responsabilidad alguna de su parte". Que el contrato pueda perder su vigencia es claramente una relativización del significado de indefinitud del nombramiento. La incertidumbre causada por esta eventualidad es manifiesta: "Entonces yo le pregunte a la persona que me lo dio a firmar [el contrato]: 'Oye, ¿iqué!?... O sea, ¿esto quiere decir que se acaba el dinero del programa y ya... bye?"'. En otro caso, esta inconveniencia se expone con cierta ironía: "Para mi la única parte triste es esa en que te dicen que si se acabaron los recursos tienen cinco días para avisarte, ¿no? [...] Porque de otra manera, no se si sepas, pero las Cátedras Conacyt son plazas definitivas [sic.]". Para algunos entrevistados, incluso, la posibilidad de que el programa se vea interrumpido por razones de índole financiera es más una convicción que una contingencia: "Yo no creo que el Conacyt nos vaya a pagar diez años, así como se está haciendo ahorita”. El argumento fundamental es que siendo el programa un proyecto federal, estaría por lo tanto inevitablemente sometido a los vaivenes políticos, 
Revista de Ciencias Sociales y Humanidades. ISSN-P: 0188-9834 ISSN-E:2395-8669.

por cuya razón difícilmente sobreviviría a varias administraciones seguidas o a cambios bruscos en las prioridades de gobierno, sobre todo las derivadas de las alternancias. De cualquier manera, es palpable la angustia que produce en la mayoría. En este sentido, uno hacía la siguiente solicitud: "Que te aseguraran [...] que ya tienen contemplado ese dinero para cierto periodo. No que de pronto, a los dos años: ¿¿Qué crees? ¡se acabó!' [...] Imagínate que te avisen cinco días antes. Que ni siquiera te den chance de buscar como que otro empleo".

Pero el escenario de incertidumbre sobre la permanencia en el puesto tiene que ver también con el riesgo de ser separado por razones no del todo claras o comprendidas. Por supuesto que hay causales de despido relacionadas con las evaluaciones negativas al desempeño que son más o menos conocidas y aceptadas por todos. Y esto lo describía con fuerza un catedrático: "Las reglas del juego son meritocráticas. Tú tienes que estar produciendo. Si no, sales. Me parece que por ahí no hay mucho margen para hacerse tonto". Pero hay dos circunstancias cuyas implicaciones para la permanencia en el puesto tienen un cierto grado de ambigüedad por su relativa indefinición formal, pudiendo tornar la situación vulnerable. Una se relaciona con la ruptura del match que fuera necesario que se estableciera para que haya ocurrido el nombramiento y la correspondiente comisión. La otra tiene que ver con la naturaleza del nombramiento como "personal de confianza".

Respecto a la primera circunstancia, como se sabe, el nombramiento se produce si existe un "empate" o match entre los intereses del aspirante y la institución a la cual se le enviaría en comisión en el horizonte de un proyecto previamente ganador. En ausencia de ese match no tendría lugar la contratación. En condiciones normales el plazo de la comisión se determinaría por las características del proyecto. ¿Qué sucedería en el caso de que finalmente, por alguna razón, desaparezcan las condiciones que generaron el "empate" que a su vez dio lugar al nombramiento inicial? El quiebre de este match puede ocurrir antes de que finalice el plazo pactado de la comisión o al término natural de la misma.

Si ocurriera dentro del plazo, pero si la interrupción de la comisión ocurriera por razones atribuibles exclusivamente a la institución - ya sea porque decida renunciar al beneficio de la Cátedra (CONACYT, 2014a: 14), ya sea por falta de cumplimiento de sus compromisos (CONACYT, 2014a: 19)—, Conacyt buscará otra institución donde se requieran los servicios del catedrático. Es una situación prevista en los reglamentos y, por tanto, carece de importancia para este trabajo.

Pero una situación distinta es aquella en la que el plazo se cumple según lo pactado. De acuerdo al Artículo 25 de los Lineamientos (CONACYT, 2014a: 14) la comisión de la Cátedra Conacyt podrá⿴ ser: 1) por un plazo de hasta 10 años a partir de la fecha de contratación; (2) pero renovable las veces que sean necesarias hasta el cumplimiento de los objetivos establecidos en el proyecto seleccionado.

La comisión parece estar en función del proyecto. Suponiendo una situación "ideal" en la que el catedrático lograra permanecer sin contratiempos en la institución hasta que concluya el proyecto, entonces la comisión finalizaría, deshaciéndose con ello el match previamente establecido y probablemente deviniendo incierta la situación laboral. Para algunos, integrados en una suerte de realidad sobreentendida, no existe la más mínima duda de que es responsabilidad de Conacyt reubicarlos. Lo asumen como si fuera parte del contrato. Pero para otros, como realmente todavía no existe una formulación clara al respecto, las dudas brotan con intensidad: “... en la parte más bonita, todo mundo es feliz y todo mundo desarrolla investigación por diez años. Acabados los diez años, el gobierno tendría que renovar ese convenio, [pero] es más o menos incierto qué vaya a pasar después de diez años". En similar tenor, otro 
Revista de Ciencias Sociales y Humanidades. ISSN-P: 0188-9834 ISSN-E:2395-8669.

investigador, al explicar su punto de vista acerca de las opciones al término de la comisión, indica que una de ellas es que por cualquier motivo también finalice el convenio con la institución. Y señala que "en ese caso, yo me imagino que nos transfieren a otro lado [pero] no sé cómo funciona exactamente. Realmente, ni siquiera ellos tienen idea de cómo va a funcionar eso. Y cuando preguntamos, nos dijeron: 'Todavía no sabemos"'.

La segunda circunstancia que puede provocar separación del puesto por razones poco claras descansa en el nombramiento de los catedráticos como personal de confianza de Conacyt (CONACYT, 2014a: 8). En este mismo documento (Lineamientos) se especifican siete causales de rescisión del contrato. Seis son bastante específicas: cuatro referidas al desempeño, dos a cuestiones éticas. La séptima, sin embargo, es de carácter general, y remite a otras causales "contenidas en la legislación laboral correspondiente y demás disposiciones aplicables" (pág. 18). El punto aquí es que conforme al artículo 185 de la Ley Federal del Trabajo no es necesario alegar ninguna causa justificada de rescisión, ni siquiera una falta de integridad moral, para finalizar el contrato de un trabajador de confianza. Basta que se alegue un motivo razonable - que puede ser incluso de carácter subjetivo-, en el que se indique la pérdida de esa confianza. Constituye, en sí misma, una situación de vulnerabilidad laboral permanente, un elemento de flexibilidad que pudiera dar lugar a despidos carentes de justificación real. Resulta interesante, sin embargo, que los riesgos que objetivamente podrían derivarse de la naturaleza del nombramiento como "personal de confianza», no parece estar en el radar de sus preocupaciones. Ello es con toda probabilidad atribuible al desconocimiento general que ellos mismos alegan tener respecto de la legislación laboral, lo cual no deja de tener importancia en términos de la subjetividad.

\subsection{La doble subordinación institucional}

Los catedráticos pertenecen formalmente a Conacyt, que es quien los emplea, pero realizan sus tareas cotidianamente en y para otra institución, cuyas reglas de organización deben necesariamente obedecer o, al menos, no transgredir abiertamente. Ambas instituciones comparten responsabilidad en la situación laboral integral que afrontan los catedráticos. En efecto, derivado de los documentos que amparan su contratación, los catedráticos tienen garantizado un conjunto de condiciones generales de trabajo. Todos gozan por igual de elevados salarios e infinidad de prestaciones provistas por el contratista. Pero, en lo particular, las condiciones de trabajo van a depender también, en gran medida, de las dinámicas internas de las instituciones a las que fueron comisionados.

Trabajar en una institución a la que no se pertenece sobre una base más o menos permanente puede generar situaciones también más o menos confusas para todos los actores involucrados. De hecho, de las tres áreas de problemas examinadas, fue esta suerte de segregación laboral — patronal, espacial, de colectivos de trabajo-, aquella en la que con mayor intensidad y extensión se presentaron las contrariedades, quejas y conflictos. Hay dos puntos que consideramos importante abordar en esta parte: (1) aplicación segmentada de las reglas institucionales denotando a un trato laboral desigual y, a veces, hasta discriminatorio hacia los catedráticos; (2) confusiones en la identidad y lealtades laborales. 
Revista de Ciencias Sociales y Humanidades. ISSN-P: 0188-9834 ISSN-E:2395-8669.

\subsubsection{Reglas segmentadas: ¿trato discriminatorio a nivel institucional?}

Conacyt reconoce el significado estrictamente laboral que tienen las instituciones más allá de ser el ámbito en el que los catedráticos estarían desarrollando su quehacer académico. En el artículo 26 de los Lineamientos se especifica que las instituciones deberán asegurar que los catedráticos tengan garantizado el "acceso a la infraestructura, el equipamiento y los servicios necesarios para el normal desarrollo de sus funciones de investigación, desarrollo tecnológico e innovación, en igualdad de condiciones que el personal académico de su plantilla" (CONACYT, 2014a: 15) [subrayado nuestro]. En el mismo documento se reconoce que, además de las funciones de investigación, etc., mencionadas, también las instituciones deben garantizar la participación de los catedráticos incorporados en actividades de docencia y, en particular, en la dirección de tesis doctorales. A fin de garantizar el éxito en el cumplimiento de tal exigencia, Conacyt, por una parte, ofrece un apoyo de quinientos mil pesos para la compra de mobiliario y equipos y, por otra, obliga al mismo tiempo a las instituciones a firmar un convenio específico en el que se formalizan los derechos y obligaciones de las partes.

Sin embargo, estos mandatos no se han cumplido cabalmente en todos los casos. Algunos de los entrevistados reconocen el impulso que la institución ha dado a la actividad que realizan. Pero para otros, por el contrario, las imposiciones impuestas por las instituciones lo experimentan como un factor de freno. En general, cuatro fueron las quejas más frecuentes con relación a esta cuestión.

En primer lugar, en algunos casos las condiciones de infraestructura - de espacios, equipos, etc.- son inferiores a las de los investigadores de planta. Uno de los catedráticos planteaba al respecto: "Yo me siento, digamos, un poquito abajo de ellos. No me siento igual que un investigador de aquí [...] Precisamente ahorita estoy en un espacio más pequeño del que ellos usan, ellos tienen un espacio un poco más grande que éste". Es simbólico, pero en términos de vivencia no debe restársele importancia.

En segundo lugar, a algunos no se les permitía o se les limitaba impartir clases o dirigir tesis. Aunque no es generalizado, un caso emblemático fue el de una institución que arbitrariamente ubicó a los catedráticos en una categoría de menor rango que la que les debería corresponder en base a lo que plantean los Lineamientos. "Aquí [...] se nos da un nombramiento que, dentro de la legislación [de la institución], es en términos de profesor invitado. Y los profesores invitados no tienen todos los beneficios que tienen los profesores de planta de la institución..., ¿ ¿no? No pueden acceder a ciertos recursos, no pueden dirigir tesis - o tienen un número limitado de direcciones-... una cosa así. Es decir, el estar en un estatus especial de pronto puede significar desventajas".

En tercer lugar, no se les confían actividades de investigación como tal. La queja en este sentido es que se les trata como aprendices o asistentes, desconociendo muchas veces que llegan comisionados para trabajar en un proyecto en calidad de investigadores, aunque jóvenes, plenos. "Están contratando investigadores para que desarrollen un trabajo de investigación, ¿no? Para mí ese es el punto [...] O sea, no es que alguien me diga: ‘¡Oye!, vas a hacer esto... Y ahora hazle así o asa’. No, ¿qué pasó?... Yo sé cómo hacer lo que tengo que hacer, ¿no?”.

En cuarto lugar, se les niega el acceso a recursos institucionales extras para la investigación. Esta fue una de los descontentos más extendidos. En la mayor parte de las instituciones, no se contempla a los catedráticos dentro de los presupuestos para viajes a eventos, trabajo de campo, etc. A veces la negativa a 
Revista de Ciencias Sociales y Humanidades. ISSN-P: 0188-9834 ISSN-E:2395-8669.

estos apoyos se hace extensiva incluso a los investigadores de esas instituciones que comparten proyecto con los catedráticos. Al respecto comentaba un entrevistado que en una convocatoria en su institución para apoyos aclaraba en letras mayúsculas que "LAS PERSONAS CON CATEDRÁTICOS NO PUEDEN APLICAR A ESTAS AYUDAS”. Pero Conacyt tampoco se los provee reglamentariamente. Constituye una situación muy confusa para la mayoría. Las explicaciones, subjetivas, son de dos tipos. Por una parte, que para cubrir esa necesidad es que tienen salarios tan altos. Por otra, que ese recurso adicional no debe ser institucional, sino que debe provenir de los proyectos en los que participan.

En definitiva, los propios catedráticos consideran que son muy importantes las dinámicas institucionales. A diferencia de un trabajador normal, en su caso se multiplican los jefes y los colectivos de trabajo. Es probable que el rechazo a los catedráticos en ciertas instituciones emane, en algunos casos, de recelos por tener altas productividades; $y$, en otros, por considerarlos usurpadores, en tanto también acceden a diversos recursos institucionales aún teniendo salarios relativamente más elevados que los internos. Pero esto último son ideas que necesitarían ser corroboradas empíricamente.

Si a esto se le suma que las instituciones son sumamente diversas entre sí en sus dinámicas, es relativamente fácil entender que los desencuentros de los catedráticos pueden ser de muchos más tipos que los de cualquier otro trabajador y se pueden presentar, además, con mayor frecuencia. Una dificultad que advierten algunos de los entrevistados es que aunque Conacyt imponga convenios de compromisos obligatorios a las instituciones, no tiene la capacidad corporativa para dar seguimiento puntual a su cumplimiento. Por supuesto que un mecanismo que pudiera compensar la imposibilidad de vigilancia generalizada y permanente por parte de Conacyt, es la retroalimentación al organismo por parte de los catedráticos. En los casos más graves se ha hecho. Y con mayor o menor eficiencia, y con mayor o menor celeridad, por lo general las situaciones conflictivas se han resuelto satisfactoriamente. Pero en los casos de situaciones menos graves, los catedráticos se han abstenido de denunciar irregularidades para evitar choques y no tener que enfrentar batallas con la institución receptora que pudiera perjudicar sus evaluaciones. Tal como ha funcionado, el programa representa una especie de centralización, pero sin total concentración de poder.

\subsubsection{Identidad laboral e institucional.}

¿A quiénes los catedráticos consideran sus jefes? ¿Con cuál de las dos instituciones experimentan mayor afinidad? Es difícil una respuesta indiscutible. Para los propios entrevistados en muchos casos constituyó una apreciación confusa. Se puede decir que en el caso de los catedráticos que han enfrentado dificultades para integrarse de manera satisfactoria en las instituciones, tienden a favorecer a Conacyt como la figura principal a la cual deben responder. Por el contrario, aquellos para quienes su situación es casi mejor que soñada, tienden a identificarse más con la institución, en particular con el jefe del proyecto. Pero en casi todos los casos, sin embargo, la respuesta final atravesó un momento de dudas: "Yo me siento más Conacyt que... [...] A ver no, a ver...pérame. No, no sé... Está difícil, porque yo me siento parte de ambas, realmente... [...] No, mentiras, yo me siento más Conacyt".

La realidad es que la identidad preferida de los catedráticos no es de carácter institucional. Mas bien se expresa en torno a todo lo que representa la actividad académica — no sólo de investigación, también publicaciones, asistencia a congresos, etc. Curiosamente la docencia, a pesar de ser también una actividad 
Revista de Ciencias Sociales y Humanidades. ISSN-P: 0188-9834 ISSN-E:2395-8669.

académica en sentido estricto, no lleva la misma fuerza identitaria que las otras-, todo lo cual se traduce en proyectos de desarrollo personal altamente individualizados.

Hay, por último, otra cuestión adicional asociada a esta área de relaciones laborales triangulares que hipotéticamente supusimos importante. Nos referimos a la representación y mecanismos de protección colectiva. Se sabe que por el Artículo 183 de la Ley Federal del Trabajo que los trabajadores de confianza no podrán formar parte de los sindicatos. Lo interesante es que, independientemente de que la legislación se los impida, tampoco lo padecen. En este sentido pudiera asumirse el ámbito de Cátedras entonces como un espacio marcado no sólo por la individualización y a la vez la descolectivización de las relaciones laborales (Villagra, 2012).

A manera de conclusiones

Ante todo, debe señalarse que Cátedras es un fenómeno con apenas tres años de funcionamiento, con rasgos todavía en proceso de formación y arraigo, por lo que las conclusiones del estudio no pueden tener más que un carácter tentativo, y por ello tal vez provisional. No por ello, sin embargo, dejan de ser importantes, por sus implicaciones, para el mundo laboral correspondiente a este tipo de trabajadores.

Con esta aclaración, se pueden avanzar un conjunto de ideas que surgen directamente de los resultados del examen realizado a partir de la información de campo reunida:

Considerando el régimen de triangulación laboral que distingue a las Cátedras, se puede plantear que las mismas constituyen empleos atípicos marcados con el sello de la flexibilidad laboral. Si bien reúne características asociadas en la literatura a la subcontratación laboral, y en este sentido puede considerarse como una expresión o manifestación de ese mismo fenómeno, también presenta características propias, diferentes de las usualmente consideradas en su descripción.

Pero difícilmente podrían ser catalogados como empleos precarios: salarios altos, prestaciones casi cabales, protección contractual, el elevado grado de satisfacción esencial como experiencia laboral subjetiva, etc., amparan esta consideración. Igualmente, por razones tanto teóricas como metodológicas, tampoco cabría hablar de algún "grado" de precariedad, en el sentido planteado por Rodgers. Tal vez, sí, podría pensarse en áreas precarias (permanencia del empleo, defensa colectiva), siguiendo más la lógica de Bouffartigue. Por tanto, la idea bastante extendida en el sentido de que la subcontratación y, en especial, la triangulación laboral, desencadenan procesos precarios, no parece obtener pleno respaldo en los hallazgos de esta investigación.

A pesar de esta satisfacción general hay, no obstante, un conjunto de cuestiones con relación a las cuales existe un cierto nivel de descontento e inconformidad. Podría decirse que Cátedras constituye un espacio de autonomía laboral favorable que no obstante se ha venido desarrollado sobre un fondo de incertidumbre. La mayoría de las problemáticas experimentadas, sin embargo, han sido circunstancias que por lo novedoso del programa no fueron siquiera previstas. Algunas se han hecho manifiestas, y como regla se han enfrentado y solucionado satisfactoriamente, a pesar de la falta de experiencia de Conacyt al respecto. Constituyen situaciones que a medida que se sigan suscitando con toda seguridad irán contribuyendo a incrementar el acervo de saberes institucionales para poder atenderlas con mayor eficiencia en el futuro. Hay otras que se mantienen latentes o son de manejo más complejo. La atención, por ejemplo, a los elementos que conllevan a incertidumbre respecto de la permanencia en el puesto, o sobre los mecanismos que garanticen por parte de las instituciones los convenios suscritos, son cuestiones que no deben ser descuidadas. 
Revista de Ciencias Sociales y Humanidades. ISSN-P: 0188-9834 ISSN-E:2395-8669.

Considerando integralmente las experiencias presentadas por los catedráticos pudiera sintéticamente plantearse que las Cátedras Conacyt constituyen, en palabras prestadas de Reygadas "empleos que presentan limitaciones"; pero al mismo tiempo sin dejar de constituir esencialmente empleos de calidad.

\section{Referencias}

Acosta, Irma y Claudia Aguilar. 2014. La situación laboral en la academia: asimetrías por género en nivel de posgrado de la Universidad Autónoma de Zacatecas. En Género, salud y condiciones de trabajo en la ciencia, editado por Veronika Sieglin y Irma Acosta. México, D.F.: AM Editores / Clave Editorial, 170-213. Añez, Carmen. 2012. Subcontratación y triangulación laboral: relaciones encubiertas. Revista Venezolana de Análisis de Coyuntura, 18 (2): 163-177.

Arce, Oyuki. 2016. Cátedras Conacyt: ¿precariedad laboral o nuevas formas de contratación? Maestría en Estudios de Población, Tijuana: El Colegio de la Frontera Norte.

Bensusán, Graciela. 2007. La subcontratación laboral y sus consecuencias: ¿problemas de diseño institucional o de implementación? En Propuestas para una reforma laboral democrática, editado por Alfonso Bouzas Ortiz. Ciudad de México: Universidad Nacional Autónoma de México, 129-159.

Bouffartigue, Paul. 2008. Précarités professionnelles et action collective: La forme syndicale à lépreuve. Travail et emploi, (116): 33-43. DOI: https://doi.org/10.4000/travailemploi.4045.

Bronstein, Arturo. 1990. La flexibilidad del trabajo: panorama general. Revista de la Facultad de Ciencias Jurídicas y Políticas, 35(75): 372-413.

Cano, Ernest. 1998. La lógica de la precariedad laboral: El caso de la industria valenciana del mueble. Cuadernos de Relaciones Laborales, 13: 207-227.

CONACYT. 2014a. Lineamientos para la Administración de las Cátedras CONACyT. Consejo Nacional de Ciencia y Tecnología (Conacyt). http://www.conacyt.mx/index.php/el-conacyt/convocatorias-yresultados-conacyt/convocatoria-catedras/convocatorias-catedras-conacyt-para-jovenes-investigadores-2016/10806-lineamientos-de-catedras-conacyt/file (11 de abril, 2016).

. 2014b. Convocatoria para jóvenes investigadores, 2014. Consejo Nacional de Ciencia y Tecnología (Conacyt). http://www.conacyt.mx/index.php/el-conacyt/convocatorias-y-resultados-conacyt/convocatoria-catedras/convocatoria-2014-3/convocatorias-para-jovenes/9101-convocatoria-investigadores-2014/file (8 de diciembre, 2015).

2014c. Convocatoria para Instituciones de Adscripción, 2014. Consejo Nacional de Ciencia y Tecnología (Conacyt). http://www.conacyt.mx/index.php/resultados-extranjero/convocatoriasconacyt/convocatoria-catedras/convocatoria-2014-3/convocatoria-instituciones/9097-convocatoriainstituciones/file (5 de julio, 2016).

Curiel, Verónica. 2013. La reforma a la Ley Federal del Trabajo en materia de subcontratación en México. Alegatos, 83: 213-236.

Dean, Matteo. 2007. Outsourcing en México. Ciudad de México: Centro de Investigación Laboral y Asesoría Sindical. https://matteodean.files.wordpress.com/2007/04/oustorcing_10_pdf.pdf (11 de junio, 2016).

Diana, Nicolás. 2013. La múltiple dimensión de la precariedad laboral: el caso de la administración pública en Argentina. Revista de Ciencias Sociales, (128-129): 119-136. DOI: https://doi.org/10.15517/rcs.v0i128129.8760 . 
Revista de Ciencias Sociales y Humanidades. ISSN-P: 0188-9834 ISSN-E:2395-8669.

Echeverría, Magdalena. 1997. Subcontratación de la producción y subcontratación del trabajo. Santiago de Chile: Departamento de Estudios de la Dirección del Trabajo, Dirección de Trabajo, Gobierno de Chile. http://www.dt.gob.cl/documentacion/1612/w3-article-60338.html (26 de septiembre, 2016).

2010. La historia inconclusa de la subcontratación y el relato de los trabajadores. Santia-

go de Chile: Gobierno de Chile, Dirección del Trabajo, Departamento de Estudios. http://www.dt.gob. cl/m/1620/articles-97627_recurso_1.pdf (4 de agosto, 2016).

Ermida, Oscar y Natalia Colotuzzo. 2009. Descentralización, tercerización, subcontratación. Lima: Oficina Internacional del Trabajo. http://white.lim.ilo.org/proyectoactrav/pry_rla_06_m03_spa/publicaciones/ documentos/estudio_descentralizacion_tercerizacion_subcontratacion.pdf (10 de abril, 2016).

ESOPE. 2005. Precarious employment in europe: A Comparative Study of Labour Market Related Risks in Flexible Economies. Brussels: European Commission Research On Social Sciences and Humanities. $\mathrm{ftp} / / \mathrm{ftp} . c o r d i s . e u r o p a . e u / p u b / c i t i z e n s / d o c s / k i n a 21250$ ens_final_esope.pdf (28 de octubre, 2015).

Gil Antón, Manuel. 2014. ¿'Outsourcing' en las universidades? El Universal, 29 de marzo. México, D. F. http://www.eluniversalmas.com.mx/editoriales/2014/03/69489.php (12 de agosto, 2015).

Gobierno de la República. 2013. Plan Nacional de Desarrollo 2013-2016. Diario Oficial de la Federación (DOF). http://www.dof.gob.mx/nota_detalle.php?codigo=5299465\&fecha=20/05/2013 (5 de julio, 2016). Guadarrama, Rocío, Alfredo Hualde y Silvia López. 2016. La precariedad laboral en México. Dimensiones, dinámicas y significados. Tijuana, B.C., México / México, D.F: El Colegio de la Frontera Norte / Universidad Autónoma Metropolitana (Unidad Cuajimalpa).

Llomovatte, Silvia y Judith Wischnevsky. 2012. Condiciones laborales de los docentes universitarios en los 90. Algunos resultados de investigación. Praxis Educativa, 3 (3): 15-26.

Miguélez, Fausto. 2004. La flexibilidad laboral. Trabajo, 13: 17-36.

Mora, Minor. 2012. La medición de la precariedad laboral: problemas metodológicos y alternativas de solución. Revista Trabajo, 5(9 [enero-junio]): 87-122.

OIT. 1997. Trabajo en régimen de subcontratación [Informe VI (1), Conferencia Internacional del Trabajo, 85.a reunión]. Ginebra: Oficina Internacional del Trabajo (OIT). http://staging.ilo.org/public/libdoc/ ilo/1996/96B09_23_SPAN.pdf (11 de abril, 2016).

1998. Trabajo en régimen de subcontratación [Informe V(2B), Conferencia Internacional del Trabajo, 86.a reunión]. Ginebra: OIT (Oficina Internacional del Trabajo). http://staging.ilo.org/public/ libdoc/ilo/1998/98B09_24_span.pdf (13 de abril, 2016).

2006. La relación de trabajo [Informe V (1), Conferencia Internacional del Trabajo, 95a. reunión]. Ginebra: OIT (Oficina Internacional del Trabajo). http://www.ilo.org/public/spanish/standards/ relm/ilc/ilc95/pdf/rep-v-1.pdf (13 de septiembre, 2016).

Reygadas, Luis. 2011. Trabajos atípicos, trabajos precarios: ¿dos caras de la misma moneda? En Trabajos atípicos y precarización del empleo, editado por María Pacheco, Enrique de la Garza Toledo y Luis Reygadas. México, D.F.: El Colegio de México, 21-45.

Rodgers, Gerry. 1989. Precarious work in western europe: The state of the debate. En Precarious jobs in labour market regulation: the growth of atypical employment in Western Europe, editado por Gerry Rodgers y Janine Rodgers. Brusels: International Institute for Labour Studies and Free University of Brussels, 1-16. Slaughter, Sheila y Larry Leslie. 2001. Expanding and Elaborating the Concept of Academic Capitalism. Organization, 8 (2): 154-161. DOI: https://doi.org/10.1177/1350508401082003. 